\title{
ARTEFACTS REMOVAL FROM ECG SIGNAL: DRAGONFLY OPTIMIZATION-BASED LEARNING ALGORITHM FOR NEURAL NETWORK-ENHANCED ADAPTIVE FILTERING
}

\author{
TALABATTULA VISWANADHAM *AND P RAJESH KUMAR ${ }^{\dagger}$
}

\begin{abstract}
Electrocardiogram (ECG) artefact removal is the major research topic as the pure ECG signals are an essential part of diagnosing heart-related problems. ECG signals are highly prominent to the interaction with the other signals like the Electromyography (EMG), Electroencephalography (EEG), and Electrooculography (EOG) signals and the interference mainly occurs at the time of recording. The removal of the artefacts from the ECG signal is a hectic challenge, for which, a novel algorithm is proposed in this work. The proposed method utilizes the adaptive filter termed as the (Dragonfly optimization + Levenberg Marqueret learning algorithm) DLM-based Nonlinear Autoregressive with eXogenous input (NARX) neural network for the removal of the artefacts from the ECG signals. Once the artefact signal is identified using the adaptive filter, the identified signal is subtracted from the primary signal that is composed of the ECG signal and the artefacts through an adaptive subtraction procedure. The clean signal thus obtained is used for effective diagnosis purposes, and the experimentation performed to prove the effectiveness of the proposed method proves that the proposed method obtained a maximum Signal-to-noise ratio (SNR) of $52.8789 \mathrm{~dB}$, a minimum error of 0.1832 , and minimum error of 0.428 .
\end{abstract}

Key words: ECG artefact removal, Dragonfly optimization, LM algorithm, NARX neural network, adaptive filter.

1. Introduction. With a lot of technical advances, the people of the world are afflicted with chronic diseases, among which the cardiovascular diseases are most commonly available diseases all over the world. The process of treatment and diagnosis of cardiovascular disease needs continuous monitoring with extensive care, but due to the increase in the number of patients, it is a tough task to undergo continuous monitoring of the patients that imposes the need for remote health monitoring. The remote health monitoring gains remarkable importance that involves the process of monitoring the ECG signals of the patient from a remote area by placing a mobile device in the patient's body during the normal day to day activity of the patient [17]. ECG is the graph that represents the electrical conducting system of the heart that lies in the value range of $\pm 2 \mathrm{mV}$ and bandwidth range of $0.05 \mathrm{~Hz}$ to $125 \mathrm{~Hz}$ [1]. Thus, ECG consists of three waves, namely $\mathrm{P}$ wave, T wave, and $\mathrm{U}$ wave along with a QRS complex, and these are used for diagnosing the cardiac diseases [19]. The ECG has a lot of advantages not only in diagnosing the cardiac diseases but also in treating the obstructive sleep apnea or wearable physiological monitor and in checking the efficiency of the therapeutic drugs [19] [20]. The major issue is that the ECG signals are affected by the presence of noise, such as power line interference, motion artifacts, electromyogram effects, and baseline drift with respiration [3].

The ECG signal is affected by various kinds of artefacts at the time of the acquisition in the clinical atmosphere. The artefacts that affect the ECG in the clinical atmosphere include the baseline wander (BW), power-line interference (PLI), muscle artifacts (MA), and motion artifacts [4]. These artefacts are added in the ECG during the time of the recording [1] and the various noises present in the ECG affects the diagnosis procedures, which poses the necessity for a separation of the ECG signals from the midst of the artefact for the purpose of simple interpretation [18]. The artefacts have a lot of impact on the ST segment, decrease the quality of the ECG signal, and degrade the frequency resolution and, in turn, generate signals of large amplitude in ECG that appears like the PQRST waveforms. Moreover, the artefacts hide the tiny features that are essential for diagnosis and clinical monitoring. The main objective is regarding the removal of these artifacts that enable an artefact-free ECG for proper diagnosis. The main problem is regarding the separation of the high-resolution

\footnotetext{
*Aditya Institute of Technology and Management, Tekkali, K Kotturu, Andhra Pradesh 532201, India (talabattula viswanadham@gmail.com).

${ }^{\dagger}$ Andhra University college of engineering Visakhapatnam, Andhra Pradesh, 530003, India
} 
ECG signals from the recorded ECG that is affected by the background noise [4]. The normal ECG signal has a predictable direction, duration, and amplitude of the characteristic waves, and an ECG signal is said to be normal or abnormal by assessing the ECG [1]. The ECG with the artefacts can be used for diagnosis by an experienced cardiologist, but the ECG analyzer can yield better accuracy. However, removal of the artefacts from the ECG signal enables accurate and simple interpretation [2].

The removal of noise from the ECG signal is performed using a number of the traditional algorithms that use any of the techniques, namely spatial or temporal averaging techniques. Initially, the noise is considered as random and stationary, and the noise reduction using the temporal averaging is the mean value of the frames or beats [9], and this method needs a number of the time frames for the reduction of the noise. At the same time, the spatial averaging method suffers from the problem of placing a number of electrodes in the same physical position. Along with the linear noise filtering method, the adaptive filtering methods have been used for separating and identifying the component waves of the ECG from the noisy ECG. The parameter of the filter is synchronized with the period of the signal for generating the quasi-periodic pattern of the cardiac signal. The other methods for ECG artefact removal are the subspace rotations, neural networks, and bi-spectral analysis [6]. Ensemble Averaging (EA) is the other familiar method that extracts the required components from the noisy ECG signal that averages the beats but loses the significant variations of the inter-beat in the cardiac cycle due to the averaging procedure [10] [14]. The optimization techniques [33-36] have applications in ECG artefact removal.

This paper proposes a novel algorithm for training the NARX neural network that is based on the proposed DLM optimization-based algorithm. The main intention of the paper is to remove the artefact present in the ECG signal for the effective diagnosis of cardiac diseases and other related problems. The paper presents the artefact cancelation strategy that is based on the adaptive subtraction procedure in which the artefact signal is filtered using the adaptive filter and subtracted from the artefact ECG signal. The adaptive filter uses the NARX neural network that uses the DLM optimization algorithm for tuning the weights of the network, and the effective tuning is brought about using the proposed algorithm. The proposed algorithm trains the network effectively based on the weights that correspond to the minimum value of the error. Thus, the proposed method of artefact removal stands as an effective procedure in eliminating the noise signals, such as ECG, EMG, and EOG.

The main contribution of the paper is the DLM optimization Algorithm, which determines the optimal weight for tuning the NARX neural network that serves as an adaptive filter in removing the artefacts from the ECG signal. DLM is the integration of the LM and Dragonfly optimization algorithm.

The organization of the paper is: Section 1 introduces the paper, and section 2 describes the literature works with the challenges. The proposed method is introduced and described clearly in section 3 , section 4 presents the results and discussion of the paper, and section 5 concludes the paper.

\section{Literature Review.}

2.1. NARX Neural Network. Payam Amani et al. [29] introduced a multi-step ahead response time predictor for database queries depends on a nonlinear autoregressive neural network model with exogenous inputs. The experimentation was performed to analyze the performance of the predictor on a lab setup with a MySQL-server. Zina Boussaada et al. [30] developed a race sailboat using exclusively renewable sources. It predicted the direct solar radiation on a horizontal surface using a NARX neural network. The experimental results have shown that the prediction performance was best when the training phase of the neural network is performed at regular intervals.

Eugen Diaconescu [31] tested the performance of the prediction for diverse time series using a NARX dynamic recurrent neural network (RNN). The author utilized conventional statistical techniques to occur indications to make efficient the process of prediction chaotic time series with RNN. Hong He et al. [32] introduced an ECG measuring experiment at seven acupoints of the Pericardium Meridian of Hand-Jueyin to attain the meridian information transmission data. Here, a NARX network was used to model the meridian information transmission system.

2.2. Artefact removal in ECG signal. Here, eight research works in artefact removal in ECG signal are discussed. Syed Anas Imtiaz et al. The paper [1] proposed a method for the automatic artefact removal 
using the Automatic artefact identification algorithm that is based on three parameters, namely the quality, interpretation quality, and computational complexity to find the absolute best use of the data and help the medical professionals in diagnosis. The method provides reliable data, but the presence of the low SNR artefacts that occur as a result of breathing interrupts the incoming waveform leading to the incapability of the filter to rectify the signal. Amit Kumar and Mandeep Singh [2] proposed a method Short Time Fourier Transform (STFT) for the decomposition of the artefacts from the ECG signal through the optimal selection of the wavelets thus, sustaining the diagnostic information. The method is highly robust and capable of removing the artefacts from the noisy physiological and non-stationary ECG Signals, but the method yielded Poor Percentage Root Mean Square Difference (PRD). Shing-Hong Liu et al. [3] proposed a method for the removal of the artefacts that initially computes the acceleration signal of the vibration using an accelerometer that is taken as a reference in the adaptive filter. Finally, the Least Mean Square (LMS) algorithm is employed for determining the optimal weight of the filter. The method possesses a stable convergence irrespective of the level of the noise, but the performance is found to degrade if the redundant signals and the reference signals are similar.

Muhammad Zia Ur Rahman et al. [4] used an efficient sign based normalized adaptive filter named Computationally efficient adaptive filtering technique, and it possesses weight update loops for removing the artefacts from the ECG. The method efficiently removes the non-stationary noise but used only for wireless biotelemetry ECG systems. Jinseok Lee et al. The paper [5] presented a real-time method for identifying and removing the artefacts from the ECG using the Empirical mode decomposition (EMD). There are two approaches, among which the first one uses first-order intrinsic mode function (F-IMF) of EMD, and the second approach uses the three statistical measures on the F-IMF time series for measuring the characteristics of randomness and variability. The method offers proper robustness, but it suffers from Segment disconnectivity if the detected corrupted segment is not utilized for the Atrial Fibrillation (AF) detection, which, in turn, results in performance degradation.

Taigang He et al. [6] use the Independent component analysis (ICA) for detecting and removing the artefacts from the ECG's, and the advantages of the method is that the method offers simplicity, efficiency, and hence potential for processing the ECG online using the ICA is better, but the ECG still contains the artefacts that provide the artefactual data. The method failed to remove the artefacts properly. Mbachu C.B et al. [7] proposed a method for filtering the artefacts from ECG using a method named as the Rectangular Window-Based Digital Filters. Initially, the digital finite impulse response (FIR) low pass, high pass, and notch filters are designed based on the rectangular window. The advantage is that the filters are able to remove the unwanted signals and thus, minimizes the power line interference, but distortions are present due to cascade filtering output signal that generates ripples due to the usage of rectangular windows. Guang Zhang et al. [8] designed an enhanced Least mean-square (LMS) method for degrading Cardiopulmonary resuscitation (CPR) artefacts causing reliable discovery of the VF rhythm during the uninterrupted chest compression (CC). This method reduces the CPR artefacts effectively from the corrupted ECG signal, but the performance of the enhanced LMS method is poor, and a large amount of real corrupted ECG records are required for enhancing the performance.

\subsection{Challenges.}

1. During the overlapping of the spectral content with the ECG, the SNR is enhanced using digital filtering, but this digital filtering injects little distortion in the ST-segment regions. In almost all the situations, the shape of the component wave that is present in the noise signal is well-known but the requirement is understand the time of occurrence and the exact shape of the signal [9].

2. The filters used for removal of the artefacts from the ECG signal cause a reduction in the amplitudes of the component waves [6], and they are not successful. Moreover, some of the noise and artefacts possess a wide range of the frequency, and they are random in nature; thus, filters are not successful in eliminating the interference while it lies in the same frequency range of the cardiac signal.

3. The major and significant feature regarding the ECG waveform is the QRS complex that lies in a particular frequency band. The relative power of the wave requires a specific examination that ensures the reliability of the ECG waveform. The value of the PSQI defines the presence, or the absence of the data of interest, which in other words, can be briefed as the presence of the higher value of pSQI indicates the required data is present or else the required data is missing. The strict monitoring should 
be enabled as the artefacts caused as a result of breathing, muscle contractions, and general body motion possess a frequency of $5 \mathrm{~Hz}[1]$.

4. The LMS adaptive filtering is used in [4-8] for the dismissal of the artefacts from the ECG signal, and this method is the old algorithm that uses the predefined reference signal to remove the artefacts efficiently.

5. The monitoring tools namely, EOG, EMG, and EEG are involved in the process of continuous monitoring along with the ECG that causes the rhythmic artefacts to be included in the mechanical activity of the ECG. The continuous monitoring process causes the interference of the EOG, EMG, and EEG signals in the ECG such that the removal of artefacts remains the better way for proper diagnosis [8].

3. The proposed method for the ECG artefact removal. The reason to remove the artefacts from the ECG signals is performed using the newly proposed DLM optimization-based NARX neural network. The paper gives a brief discussion of the proposed method of artefact removal from the ECG signals that gains a lot of advantages like an effective diagnosis of cardiac diseases and helps the physician to take effective measures.

3.1. Removal of artefacts from the ECG signals. ECG is the record of the electrical activity of the heart, and during the recording process, the artefacts are included with the ECG signals due to the interference effects of the other signals, such as EEG, EOG, and EMG that may create adverse effects on the diagnosis of the cardiac-related diseases. In order to overcome the problems caused by the artefacts, it is essential to perform the adaptive noise cancelation strategy [24] for the removal of the artefacts, such as EEG, EOG, and EMG signals. Thus, to get rid of the artefacts using the adaptive noise cancelation, the cancelation framework requires two inputs. Among two of the inputs, one input comes from the ECG signal source, whereas the second input is from the artefacts. Thus, the primary input signal is the combination of the signal from the ECG source, which is the clean signal and the interference signal that is obtained by passing the artefact through the unidentified non-linear dynamics. Thus, the primary signal is represented as

$$
B(i)=C(i)+I(i)
$$

where $B(i)$ is the primary input signal, $C(i)$ refers to the clean ECG signal, and $I(i)$ stands for the interference that is generated using the unknown nonlinear dynamics or otherwise the signal obtained using the noise source. The noise signal is subjective to adaptive filtering to produce the filtered output, which is similar to that of the interference signal created as a result of the nonlinear dynamics. Thus, the noise cancelation is performed that extracts the clean signal through the subtraction of the filtered output from the primary input signal. Thus, the clean signal extracted using the noise cancelation strategy is given as

$$
C^{*}(i)=B(i)-A(i)
$$

where $C^{*}(i)$ indicates the clean signal obtained as a result of the adaptive noise cancelation, $B(i)$ stands for the primary input signal, and $A(i)$ refers to the adaptive filtered output. Figure 3.1 shows the proposed noise cancelation strategy.

3.2. NARX neural network for the enhanced adaptive filtering of the artefacts from the ECG signal. The proposed method of the artefact removal from the ECG signals using the NARX neural network is presented in this section. The main aim of the NARX neural network is to predict the artefact present in the ECG signal to generate the clean ECG signal sufficient for perfect diagnosis. The input to the neural network is the artefact and the signal is predicted for which the delays are used.

3.2.1. Solution Encoding. Figure 3.2 shows the solution of the proposed learning algorithm that yields an optimized solution using three weights, namely the weights of the exogenous input vector, weights of the regressed output vector, and the weights of the exogenous output vector. These three weights are combined to generate an optimized weight such that the optimized output trains the NARX neural network in canceling the artefacts and the size of the solution vector depends on the number of hidden neurons present in the network. Let us consider the weights of the exogenous input vector as $L_{1}, L_{2}, L_{d 1}$, the weights of the regressed output is represented as $R_{1}, R_{2}, R_{d 2}$, and the exogenous output vector as, $O_{1}, O_{2}, O_{d 1}$. Then, the solution generated using the LM algorithm, and the Dragonfly optimization algorithm is represented as $X^{l 1}, X^{l 2}, X^{l f}$ and $X^{d 1}, X^{d 2}, X^{d f}$ respectively. Thus, the proposed DLM generated the optimal weights that is given as $X^{z 1}, X^{z 2}, X^{z f}$. 


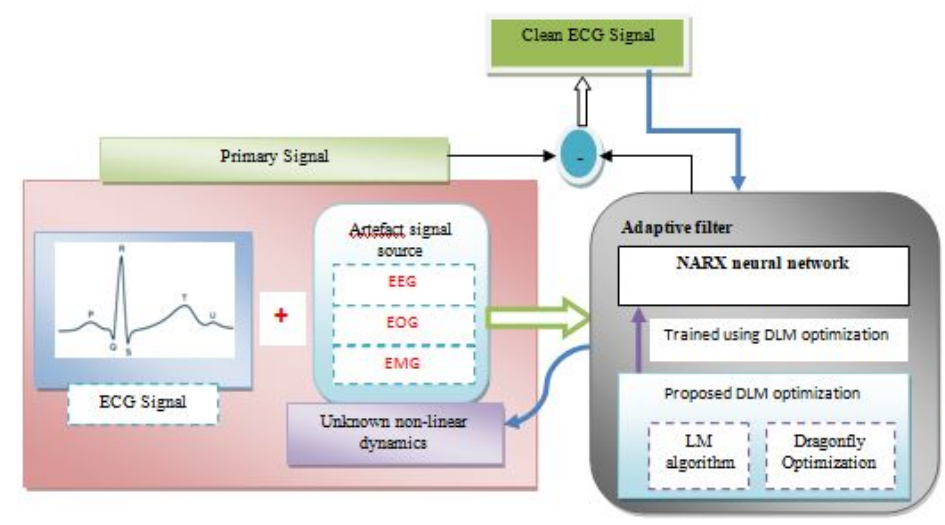

FIG. 3.1. ECG artefact removal

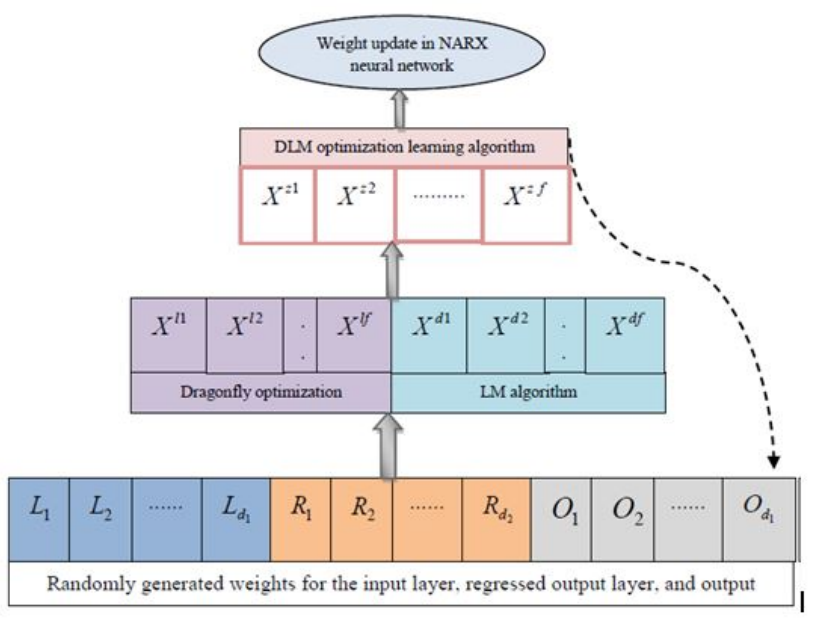

FIG. 3.2. Solution encoding of the DLM optimization-based NARX neural network

3.2.2. Architecture of the NARX neural network. NARX neural network [22] is a recurrent neural network that is used for the analysis and modelling of the nonlinear time series and holds a lot of merits when compared with the other classical prediction models. The NARX network possesses an effective learning rate, and in the proposed method of noise cancelation, the learning algorithm used is the dragonfly optimization and the LM algorithm. The NARX neural network is the collection of the multilayer fed forward network, recurrent loop, and the time delay. Figure 3.3 shows the architecture of the NARX neural network. The three layers include the input layer, the hidden layer, and the output layer. The network is subjected to the tapped delays both in the input layer and the output layer and the feedback flows in a single direction. The feedback follows the input layer, hidden layer, and provides the output in the output layer. There are three information vectors in the input layer namely, the exogenous input vector, delayed regressed output vector, and the delayed exogenous input vector. The output of the NARX neural network is given by

$$
N(l+1)=F\left[N(l), N(l-1), N(l-2) \ldots, N\left(l-d_{1}\right) ; S(l), S(l-1), S(l-2) . ., S\left(l-d_{2}\right)\right]
$$

where $N(l)$ is the exogenous input vector, $N(l-1), N(l-2)$., $N\left(l-d_{1}\right)$ are the delayed regressed output vector, and $S(l), S(l-1), S(l-2) ., S\left(l-d_{2}\right)$ are the delayed exogenous input vectors. At the beginning of the NARX network function, the weights are assigned between the input layer and the hidden layer and the regressed output vector and the hidden layer. 


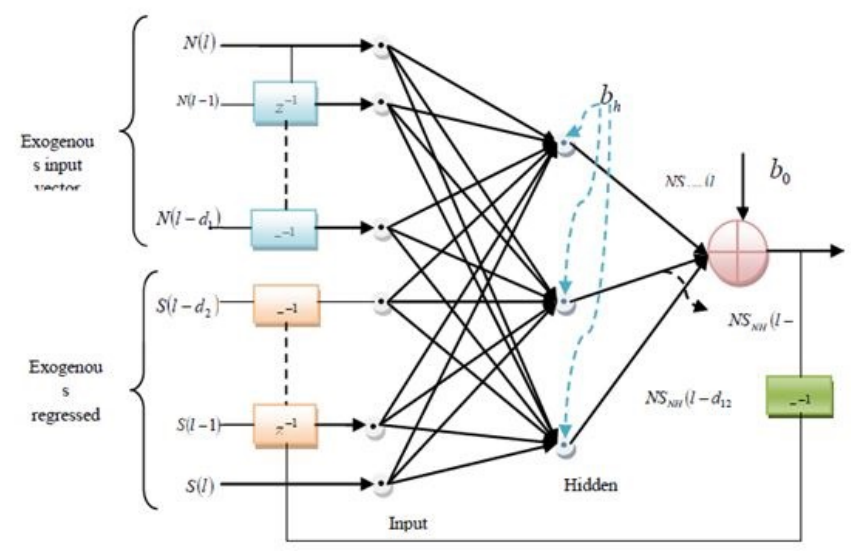

FIG. 3.3. The architecture of the NARX neural network used for adaptive filtering of the artefacts

3.2.3. A novel learning algorithm for the NARX neural network: - Proposed DLM-Dragonfly Levenberg Marqueret optimization-based Learning algorithm. The paper proposes a new learning algorithm using dragonfly optimization [21] and the LM algorithm [23]. The dragonfly optimization is the meta-heuristic optimization algorithm that holds better among most of the evolutionary algorithms because of the following reasons. The main advantage is regarding the search space as all the information regarding the search space is restored, and there are only very few control parameters in the search space leading to the increase in the flexibility of the algorithm. The proposed algorithm is robust, and the problem of converging to the local minimum using the LM algorithm is solved using the Dragonfly that increases the convergence and converges to the global minimum. The proposed algorithm is advantageous as they utilize the advantages of both the LM and Dragonfly. The learning algorithms update the weights individually, and the error values are computed for both the algorithms. The learning algorithm with the minimum value of the error is used for updating the weights of the NARX neural network, and the following are the algorithmic steps of the proposed DLM optimization-based learning algorithm.

Step 1: Population Initialization. The initial step is the initialization that initializes the total swarm population that aims at the possibility of survival. The population initialization using the dragonfly optimization is represented as

$$
D_{d} ;(1 \leq d \leq n)
$$

where $D_{d}$ denotes the position of the $d^{\text {th }}$ dragonfly and $n$ is the total population of the dragonflies.

Step 2: Parameters influencing the position update. The dragon population aims at the possible location to live as they move in search of food, and they are distracted from the enemies that remain the two basic behaviours of the swarm population. The position update follows the initialization step, and the position of the dragonflies is updated based on five major factors, separation, alignment, cohesion, attraction, and distraction, and they are based on the swarm behaviours of survival. The swarm behaviour explains the factors as the separation is the factor that avoids collision among the individual dragonflies whereas, an alignment that corresponds to the velocity matching among the dragonflies. The parameter termed as collision aligns the dragonfly towards the center of mass. The five factors are modelled as

$$
S_{d}=-\sum_{j=1}^{N} D-D_{j}
$$

where $D$ is the current position of the individual dragonfly, $D_{j}$ is the position of the $j^{\text {th }}$ neighbor, and $\mathrm{N}$ be number of neighboring dragonflies with respect to the reference dragonfly. $S_{d}$ denote the separation factor of 
the individual dragonflies.

$$
G_{d}=\frac{\sum_{j=1}^{N} V_{j}}{N}
$$

where $G_{d}$ refers to the alignment of the $d^{\text {th }}$ dragonfly and $V_{j}$ is the velocity of the $j^{\text {th }}$ neighbor.

$$
C_{d}=\frac{\sum_{j=1}^{N} D_{j}}{N}-D
$$

where $D$ is the current position of the individual dragonfly and $C_{d}$ refers to the cohesion of the $d^{\text {th }}$ dragonfly.

Step 3: Define the step vectors. The position of the dragonfly is updated based on the step vector, and the main role of the step vector is to show the direction of the dragonfly, and it is employed for the larger dimensions.

$$
\Delta D_{l+1}=\left(a_{1} S_{d}+a_{2} G_{d}+a_{3} C_{d}+a_{4} M_{d}+a_{5} \varepsilon_{d}\right)+w \Delta D_{l}
$$

where $a_{1}, a_{2}, a_{3}, a_{4}$, and $a_{5}$, are the weights of separation, alignment, cohesion, food factor, and enemy factor respectively. The values of the weights play a major role in the transition of the exploration phase and the exploitation phase of the dragonfly thus, the weights are tuned such that it ensures proper switch off between the two phases. $W$ denotes the initial weight, represents the iteration number. $s_{d}, G_{d}, C_{d}, M_{d}$, and $\varepsilon_{d}$ denotes the separation, alignment, cohesion, attraction, and distraction of the $d^{\text {th }}$ dragonfly. The exploration phase of the dragonflies describes the hunting mechanism of the dragonfly for which it takes a back and forth movement. The exploration phase describes the grouping and the movement of the dragonflies in the same direction for a long distance.

Step 4: Compute the objective function of the Fireflies. The objective function is calculated to determine the optimal weight that is based on the minimum value of the error calculated using the LM algorithm and the Dragonfly optimization algorithm. The weight corresponding to the minimum error is selected as the weight vector to train the network.

Step 5: Update and determine the position of the food source and the enemy. The above discussion highlights the behaviour of the dragonflies and hence, it is essential to update the position of the enemy and the position of the food. The computation of the position of the food and the position of the enemy is performed as

$$
M_{d}=D^{*}-D
$$

where $M_{d}$ represents the attraction of the dragonfly towards the food, $D^{*}$ is the position of the food

$$
D_{l}^{d}=D^{-}+D
$$

$Y^{-}$is the position of the enemy, and $D_{l}^{d}$ is the distraction of the dragonfly away from the enemy.

Step 6: Determine the position of the dragonfly. The position update of the dragonfly using the step vector is formulated as

$$
D_{l+1}^{d}=D_{l}^{d}+\Delta D_{d}^{l+1}
$$

Moreover, the random walk is the search mechanism that is utilized to perform the search process in the absence of the neighbouring dragonfly. The position update of the dragonfly is computed as the below equation.

$$
\begin{gathered}
D_{l+1}^{d}=D_{l}^{d}+\operatorname{levy}(H) * D_{l}^{d} \\
\operatorname{Levy}(x)=0.01 * \frac{r_{1} * \sigma}{|r 2|^{\frac{1}{\alpha}}} \\
\sigma=\left(\frac{\Gamma(1+\alpha) \times \operatorname{Sin}\left(\frac{\pi \alpha}{2}\right)}{\Gamma\left(\frac{1+\alpha}{2}\right) \times \alpha \times 2 \times \times\left(\frac{\alpha-1}{2}\right)}\right)^{\frac{1}{\alpha}}
\end{gathered}
$$

where $\Gamma(x)=(x-1) 1 . T$ represents the current iteration, $H$ is the dimension of the position vectors. $r_{1}$ and $r_{2}$ are the random vectors, $\alpha$ is the constant, and the value is 1.5. The optimal position of the dragonfly enables the optimal selection of the weights for training the NARX neural network. 
Step 7: Weight update based on the dragonfly algorithm. The above steps are repeated for the maximum number of iterations, and upon the application of the firefly algorithm, the input vector and the updated weights are combined as represented below

$$
X^{d}=\left(W, B^{n e w}\right)
$$

where $W$ refers to the input vector and $B^{\text {new }}$ stands for the weight updated using the dragonfly optimization algorithm.

Step 8: Calculation of the Mean Square Error. The MSE is computed between the target value and the current value computed using the dragonfly optimization.

$$
e^{d}=\frac{1}{l} \sum_{j=1}^{l}\left(X_{i}^{d}-X_{i}^{g}\right)^{2}
$$

where $X_{i}^{g}$ represents the truth table of the original data and $X_{i}^{d}$ denotes the output from the dragonfly algorithm.

Step 9: Update the weights using the LM algorithm. The weights of the NARX neural network are initialized that depends on the number of the hidden layers and the weights are denoted as below

$$
X=X^{c 1}, X^{c 2} \ldots, X^{c f}
$$

where $f$ is the total number of weights initialized using the LM algorithm.

Step 10: The computation of the mean square error. It follows the following equation,

$$
E(X)=e^{T} e
$$

where $E(X)=$ denotes the performance index, $e^{T} e$ indicates the target output, and $e$ represents the expected output.

Step 11: Weight Update based on LM algorithm. The weight of the NARX neural network is updated using the LM algorithm as

$$
\Delta X=\left[J^{T} J+\gamma k\right]^{-1} * J^{T} e
$$

where $J$ is the Jacobian matrix, $J^{T}$ is the Jacobian transform matrix and $\gamma$ is the learning rate. The update in the learning rate parameter depends on the decay function $\lambda$. For the greater values of $E(X)$, the learning rate is multiplied using the rate of the decay function. Once the learning rate is determined, then the value of $E(X)$ is recomputed using the weighted function $X=X+\Delta X$ as the trail weight. Similarly, the learning rate is divided by the decay rate, whenever the function $E(X)$ decreases. Then, the incremented values of the weights is found by the formula

$$
X=X+\Delta X
$$

Step 12: Formulation of the learning rate. Whenever the performance index $E(X)$ exceeds the trail weighted function, the learning rate is updated as

$$
\gamma=\gamma * \lambda
$$

The learning rate is multiplied with the decay value to obtain the new learning rate, and the weight update is performed based on the new learning rate that follows the steps from step 8.

Whenever the performance index $E(X)$ is less than the trail weighted function, the learning rate is updated by dividing the current learning rate by the decay function. Thus, the learning rate is represented as,

$$
\gamma=\frac{\gamma}{\lambda}
$$


Step 13: Random generation of the weights using the LM algorithm. The weight is updated using the following equation

$$
X_{l+1}^{L M}=X_{t}-[H+\mu * T]^{-1} * q
$$

where $H$ denotes the Hessian matrix of the algorithm, and it is determined by multiplying the Jacobian matrix and the transverse of the Jacobian matrix.

Step 14: Computing the gradient matrix. The Jacobian matrix is employed for determining the gradient matrix that is denoted as

$$
q=J^{T} e
$$

where $J$ represents the Jacobian matrix and e denotes the error value.

Step 15: Generated output based on the newly updated weights. The LM algorithm computes the output based on the original value of the input vector and the newly updated weights. The output is represented as

$$
X_{j}^{L M}=\left(W, B^{n e w}\right)
$$

Step 16: Re-compute the error. The error is recomputed between the output based on the newly updated weight, and the ground value, and the following formula is used.

$$
e^{L M}=\frac{1}{l} \sum_{j=1}^{l}\left(X_{j}^{L M}-X_{i}^{g}\right)
$$

where $l$ corresponds to the total number of the iterations, $X_{j}^{g}$ denotes the truth value of the input vector, $X_{L M}^{j}$ represents the output vector generated using the LM algorithm.

Step 17: Generation of the weight vector using the proposed DLM-training algorithm. The weight vectors are obtained using the proposed DLM algorithm for training the NARX neural network. The optimal generation of the weights are brought about through the proposed DLM algorithm. For the optimal selection of the weight vectors, the errors of the outputs using both the training algorithm are determined individually and the errors of the algorithms are compared. The weight vector corresponding to the low value of the error is considered for training the NARX network. When the error of the LM algorithm exceeds the error of the dragonfly optimization algorithm, then the weight vector obtained using the dragonfly optimization is selected as the optimal weights. When the error of the LM algorithm is lower than the error obtained using the dragonfly optimization, then the weight vector corresponding to the LM algorithm is used. Moreover, the error value has an impact on the damping factor, and the value of the damping factor reduces when the error of the current iteration is less than the error value of the previous iteration. Similarly, the value of the damping factor increases when the error of the present iteration is greater than the previous iteration. Thus, the error values of the dragonfly algorithm and the LM algorithm is employed for the optimal selection of the weights vectors in the DLM optimization algorithm.

$$
W_{l+1}= \begin{cases}W_{l}^{L M}+1 & \text { when }\left(e^{L M}<e^{d}\right) \\ D_{l+1}^{d} & \text { when }\left(e d<e^{L M}\right)\end{cases}
$$

The above equation represents the condition for the optimal selection of the weight vector.

3.2.4. Proposed method of the artefact removal using the DLM optimization-based NARX neural network. The main aim of the paper is depicted in Algorithm 11 that shows the steps involved in the elimination of the artefacts from the ECG signal. The ECG artefact signal comprises of the ECH signal and the artefacts, such as the EEG, EMG, and EOG signals. These artefacts are added with the ECG signal at the time of recording and monitoring and hence, removal of the artefacts is essential for the effective diagnosis. Thus, the adaptive filter is proposed that aims at the removal of the artefacts from the ECG signal, and the adaptive filter is made of NARX neural network that is trained using the DLM optimization algorithm. The proposed DLM algorithm determines the optimal weight for training the NARX neural network to perform the process of adaptive filtering. The proposed filter filters the artefact signal, and the filtered output is fed to the adaptive subtraction that causes the subtraction of the artefact from the ECG artefact signal such that a clean ECG signal is generated. 


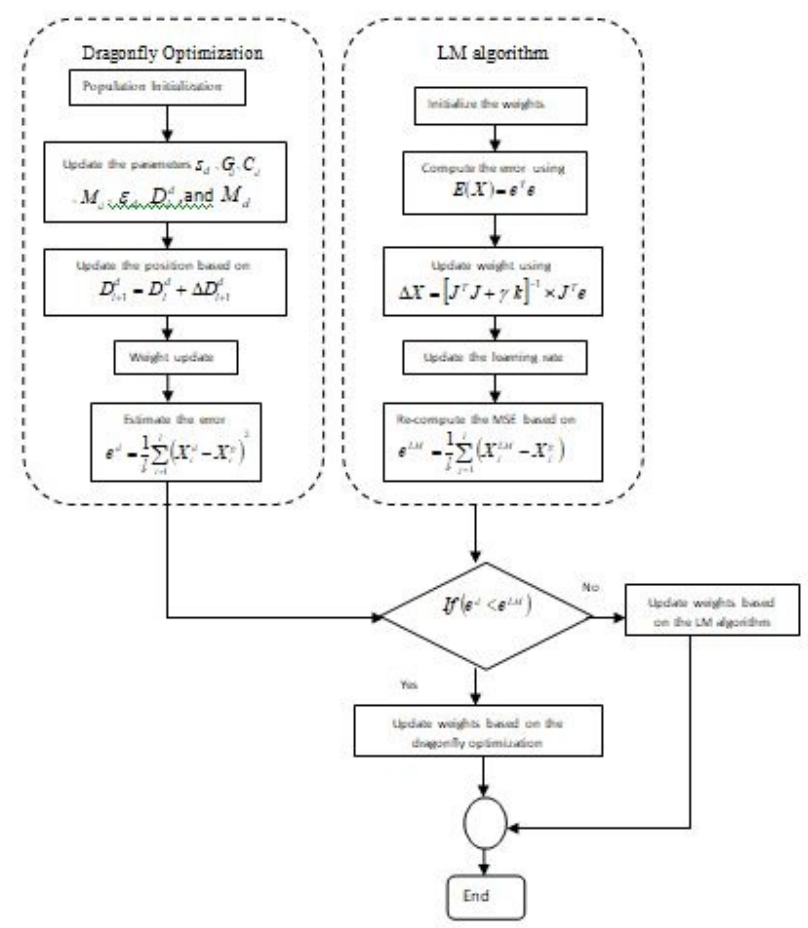

FIG. 3.4. Flowchart for the Proposed DLM optimization algorithm

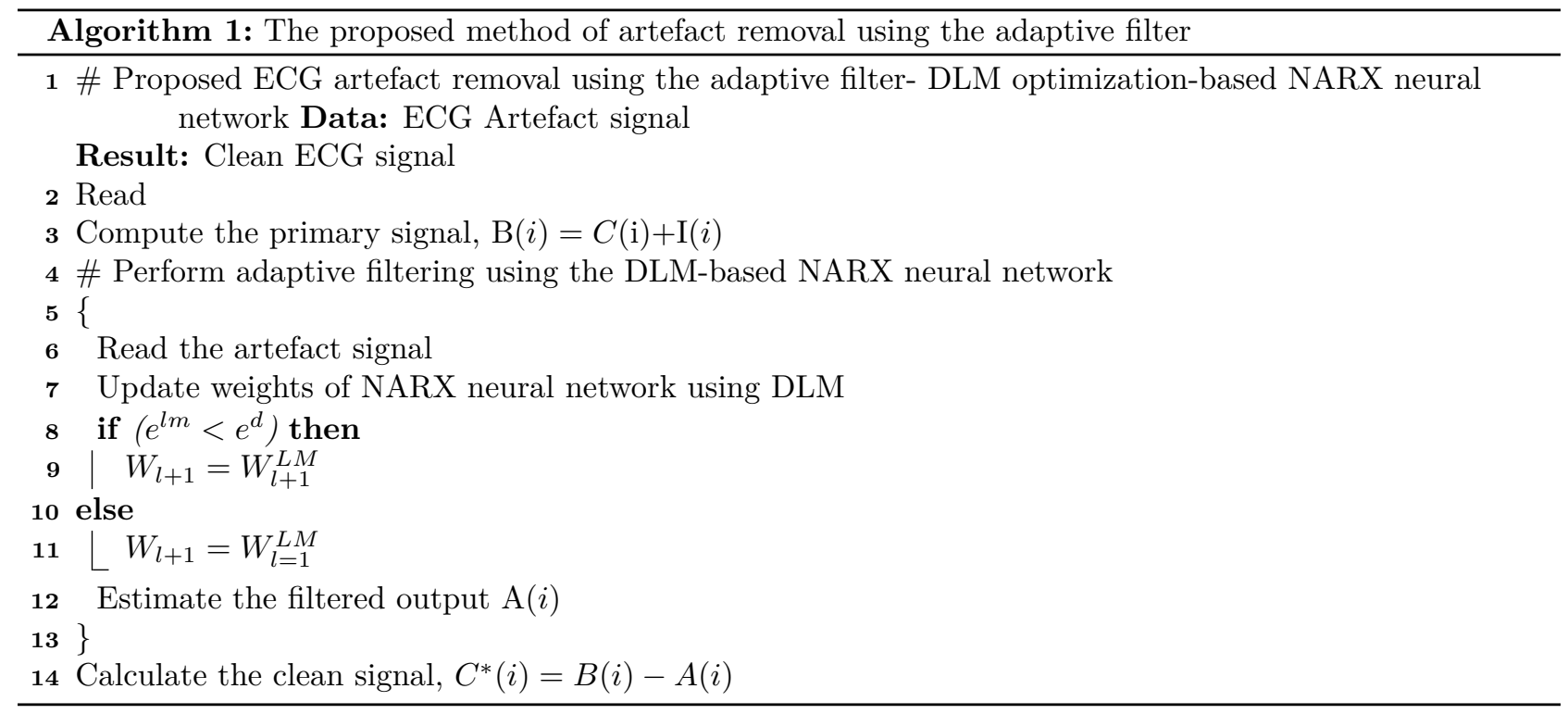

4. Results and Discussion. In this section, the result of the proposed method is discussed in detail to elaborate the superior performance of the proposed method. The experimentation of the proposed technique of artefact removal from the ECG signals is done in a system with 2 GB RAM, Intel core processor, Windows 10 Operating System. The technique is implemented using the software tool MATLAB. 


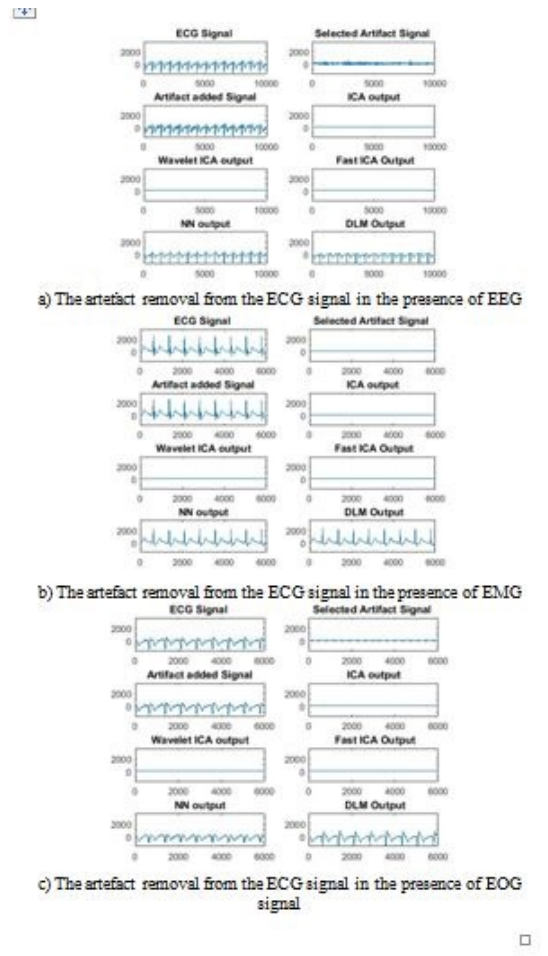

FIG. 4.1. Experimental results of the proposed method of artefact removal using various artefact removal methods

4.1. Database Description. Physionet database was created and contributed by Tatiana Lugovaya. The database has 310 ECG recordings, obtained from 90 persons. Each recording contains, ECG lead I, recorded for 20 seconds, digitized at $500 \mathrm{~Hz}$ with 12-bit resolution over a nominal $\pm 10 \mathrm{mV}$ range, 10 annotated beats, information containing age, gender, and recording date. The raw ECG signals are rather noisy and contain both high and low frequency noise components. Each record includes both raw and filtered signals: Signal 0: ECG I (raw signal), Signal 1: ECG I filtered (filtered signal).

4.2. Experimental results. Figure 4.1 shows the experimental results of the proposed method of artefact removal using various artefact removal methods. The artefacts, such as EEG, EMG, and EOG are added with the pure ECG signal during the time of recording such that the presence of artefact affects the effective decisionmaking of the doctor. Thus, the various artefact removal methods concentrate on the removal of the artefacts as is depicted in figures $4.1 \mathrm{a}, \mathrm{b}$, and c respectively.

4.3. Competing methods. The competing methods used are ICA [6], WICA [27], FICA, and NN [28] for comparing the results of the artefact removal with the proposed DLM to prove the superiority of the proposed method.

ICA: ICA is a source separation method, and its application to biomedical signals is rapidly expanding. ICA offers simplicity, efficiency, and hence the potential for processing the ECG online, but the ECG still contains the artefacts that provide the artefactual data. The method failed to remove the artefacts properly.

WICA [27]: The Wavelet-Independent Component Analysis (WICA) approach allows extending the removal of the artefacts in the clinical applications. WICA is the integration of DWT and ICA, which takes the advantages of both techniques.

FICA: Fixed point or FastICA algorithm of ICA is a technique for the removal of eye blink artifact from EEG and ECG signals. FastICA algorithm has been applied to synthetic signals prepared by adding random noise to the ECG signal. It divides the signal into two independent components, namely ECG pure and artifact signal. Similarly, it is applied to remove the artifacts from the EEG signal. 
$\mathrm{NN}$ : In [28], an adaptive filtering approach based on a discrete wavelet transform and artificial neural network is developed for ECG signal noise reduction. This method integrates the multi-resolution property of wavelet decomposition and the adaptive learning ability of artificial neural networks, and fits well with ECG signal processing applications.

4.4. Comparative analysis. Figures 4.2-4.4 show the analysis of the proposed method of artefact removal in terms of the SNR, MSE, and RMSE in the presence of the EEG, EMG, and EOG signals. Figure 4.2.a) shows the analysis in terms of SNR in the presence of an EEG signal. For the first signal, the SNR values of the methods like the ICA, WICA, FICA, NN, and DLM are $48.4474 \mathrm{~dB}, 50.9866 \mathrm{~dB}, 44.4993 \mathrm{~dB}, 47.5152$ $\mathrm{dB}$, and $50.7498 \mathrm{~dB}$ respectively. The value of SNR is $44 \mathrm{~dB}$ for ICA, $44.10 \mathrm{~dB}$ for WICA, 44 for FICA, 50.7035 for NN, and 52.8753 for DLM respectively for the second signal. It is clear that for the proposed DLM algorithm, the value of SNR is greater when compared with the existing artefact removal methods. Similarly, the analysis using the third and the fourth signal indicate that the SNR value of the proposed DLM is greater when compared with the other methods. Finally, for the fifth signal, the SNR dB's are 43.76, 44.3, 43.76, 49.83, and 51.92 for the artefact removal methods like the ICA, WICA, FICA, NN, and DLM respectively that prove the proposed method is superior over the existing methods.

Figure 4.2.b) shows the analysis in terms of MSE in the presence of an EEG signal. The effective method responds with the minimum value of the MSE error. For the first signal, the MSE values of the methods like the ICA, WICA, FICA, NN, and DLM are 20, 20, 17.5515, 0.3088, and 0.2099 respectively. The value of MSE is 15.6646 for ICA, 15.5487 for WICA, 14.3068 for FICA, 8.6930 for NN, and 4.5218 for DLM respectively for the second signal. It is clear that for the proposed DLM algorithm, the value of MSE is minimum when compared with the existing artefact removal methods. Similarly, the analysis using the third and the fourth signal indicate that the MSE value of the proposed DLM is less when compared with the other methods. Finally, for the fifth signal, the MSE values are 20, 15.4641, 15.2445, 0.8452, and 0.38 for the artefact removal methods like the ICA, WICA, FICA, NN, and DLM respectively that prove the proposed method is superior over the existing methods.

Figure 4.2.c) shows the analysis in terms of RMSE in the presence of an EEG signal. The effective method responds with the minimum value of the RMSE error. For the first signal, the RMSE values of the methods like the ICA, WICA, FICA, NN, and DLM are 4.4721, 4.4721, 4.1894, 0.5557, and 0.4582, respectively. The value of RMSE is 3.9578 for ICA, 3.9431 for WICA, 3.7824 for FICA, 2.9483 for NN, and 2.1264 for DLM, respectively for the second signal. It is clear that for the proposed DLM algorithm, the value of RMSE is minimum when compared with the existing artefact removal methods. Similarly, the analysis using the third and the fourth signal indicate that the RMSE value of the proposed DLM is less when compared with the other methods of artefact removal. Finally, for the fifth signal, the RMSE values are 4.4721, 3.9324, 3.9044, 0.9193, and 0.6165 for the artefact removal methods like the ICA, WICA, FICA, NN, and DLM respectively that prove the proposed method is superior over the existing methods.

Figure 4.3.a) shows the analysis in terms of SNR in the presence of the EMG signal. For the first signal, the SNR values of the methods like the ICA, WICA, FICA, NN, and DLM are $44.29 \mathrm{~dB}, 46.3359 \mathrm{~dB}, 44.2964$ $\mathrm{dB}, 47.1836 \mathrm{~dB}$, and $50.9222 \mathrm{~dB}$ respectively. The value of SNR is $37.9067 \mathrm{~dB}$ for ICA, 38.1276 dB for WICA, 37.9062 for FICA, 50.6968 for NN, and 52.5789 for DLM respectively for the second signal. It is clear that for the proposed DLM algorithm, the value of SNR is greater when compared with the existing artefact removal methods. Similarly, the analysis using the third and the fourth signal indicate that the SNR value of the proposed DLM is greater when compared with the other methods. Finally, for the fifth signal, the SNR dB's are 43.0636, 44.8689, 43.0636, 49.5461, and 51.939 for the artefact removal methods like the ICA, WICA, FICA, $\mathrm{NN}$, and DLM respectively that prove the proposed method is superior over the existing methods.

Figure 4.3.b) shows the analysis in terms of MSE in the presence of the EMG signal. The effective method responds with the minimum value of the MSE error. For the first signal, the MSE values of the methods like the ICA, WICA, FICA, NN, and DLM are 20, 20, 20, 84.98, and 0.192 respectively. The value of MSE is 20 for ICA, 20 for WICA, 20 for FICA, 100.8027 for NN, and 4.5206 for DLM respectively, for the second signal. It is clear that for the proposed DLM algorithm, the value of MSE is minimum when compared with the existing artefact removal methods. Similarly, the analysis using the third and the fourth signal indicate that the MSE value of the proposed DLM is less when compared with the other methods. Finally, for the fifth signal, the 


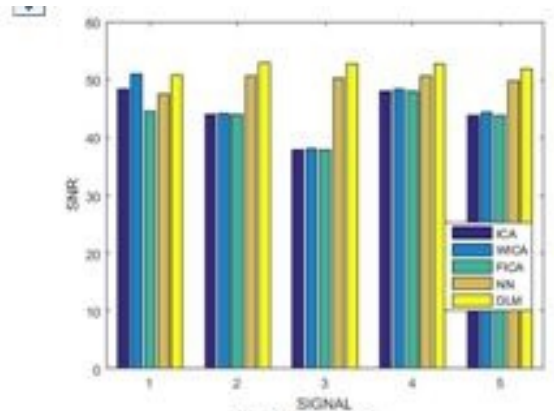

a) $S N R$ in $d B$

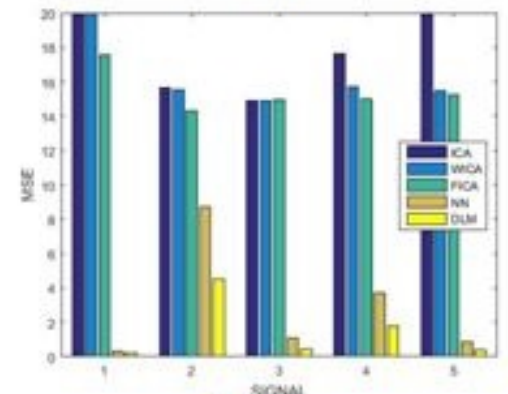

b) MSE

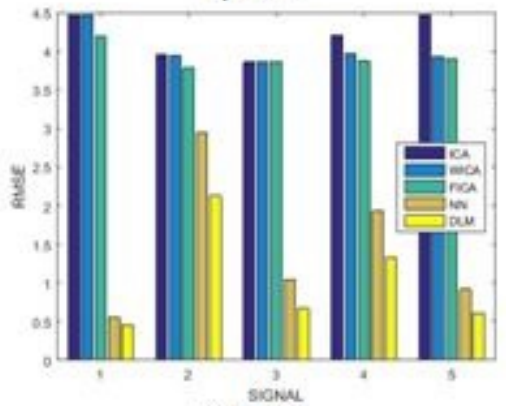

c) RMSE

FIG. 4.2. Analysis of the artefact removal in the presence of the EEG signal

MSE values are 20, 20, 12.66, 12.2077, and 0.3839 for the artefact removal methods like the ICA, WICA, FICA, $\mathrm{NN}$, and DLM respectively that prove the proposed method is superior over the existing methods.

Figure 4.3.c) shows the analysis in terms of RMSE in the presence of the EMG signal. The effective method responds with the minimum value of the RMSE error. For the first signal, the RMSE values of the methods like the ICA, WICA, FICA, NN, and DLM are 4.4721, 4.4721, 4.4721, 9.2184, and 0.4381 respectively. The value of RMSE is 4.4721 for ICA, 4.4721 for WICA, 4.4721 for FICA, 10.04 for NN, and 2.1264 for DLM, respectively for the second signal. It is clear that for the proposed DLM algorithm, the value of RMSE is minimum when compared with the existing artefact removal methods. Similarly, the analysis using the third and the fourth signal indicate that the RMSE value of the proposed DLM is less when compared with the other methods of artefact removal. Finally, for the fifth signal, the RMSE values are 4.4721, 4.4721, 3.559, 3.4939, and 0.6196 for the artefact removal methods like the ICA, WICA, FICA, NN, and DLM respectively that prove the proposed method is superior over the existing methods.

Figure 4.4.a) shows the analysis in terms of SNR in the presence of the EOG signal. For the first signal, the SNR values of the methods like the ICA, WICA, FICA, NN, and DLM are $44.2953 \mathrm{~dB}, 46.4878 \mathrm{~dB}, 44.2953$ 


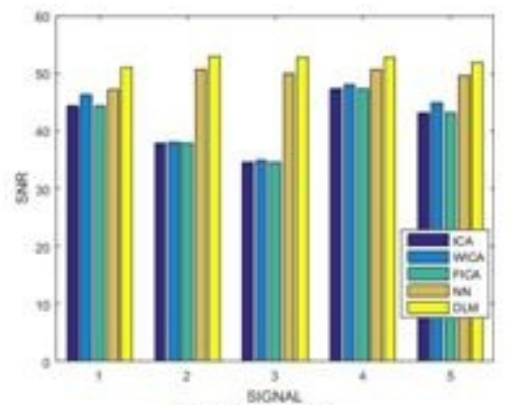

a) SNR in $d B$

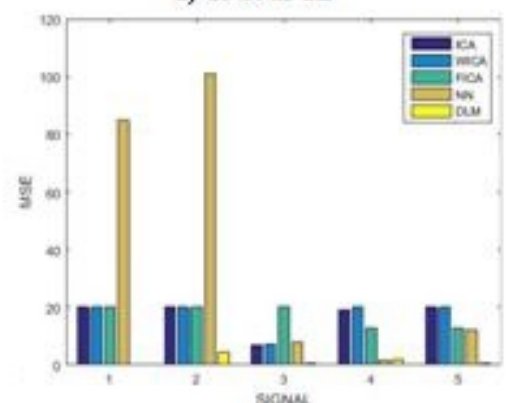

b) MSE

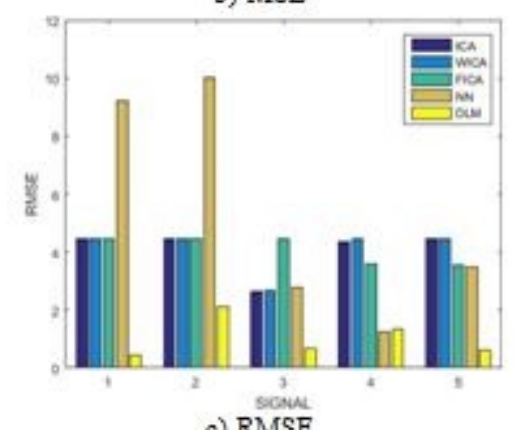

c) RMSE

FIG. 4.3. Analysis of the artefact removal in the presence of the EMG signal

$\mathrm{dB}, 48.6889 \mathrm{~dB}$, and $50.8412 \mathrm{~dB}$ respectively. The value of SNR is $37.9052 \mathrm{~dB}$ for ICA, 38.1418 dB for WICA, 37.9052 for FICA, 50.6948 for NN, and 52.8694 for DLM respectively for the second signal. It is clear that for the proposed DLM algorithm, the value of SNR is greater when compared with the existing artefact removal methods. Similarly, the analysis using the third and the fourth signal indicate that the SNR value of the proposed DLM is greater when compared with the other methods. Finally, for the fifth signal, the SNR dB's are $42.2801,43.8368,42.5828,49.4697$, and 51.8589 for the artefact removal methods like the ICA, WICA, FICA, NN, and DLM respectively that prove the proposed method is superior over the existing methods.

Figure 4.4.b) shows the analysis in terms of MSE in the presence of the EOG signal. The effective method responds with the minimum value of the MSE error. For the first signal, the MSE values of the methods like the ICA, WICA, FICA, NN, and DLM are 20, 20, 20, 116.62, and 0.1832 respectively. The value of MSE is 20 for ICA, 20 for WICA, 16.3022 for FICA, 92.5998 for NN, and 4.5254 for DLM respectively, for the second signal. It is clear that for the proposed DLM algorithm, the value of MSE is minimum when compared with the existing artefact removal methods. Similarly, the analysis using the third and the fourth signal indicate that the MSE value of the proposed DLM is less when compared with the other methods. Finally, for the fifth signal, the MSE values are 20, 20, 7.6076, 11.8829, and 0.3772 for the artefact removal methods like the 


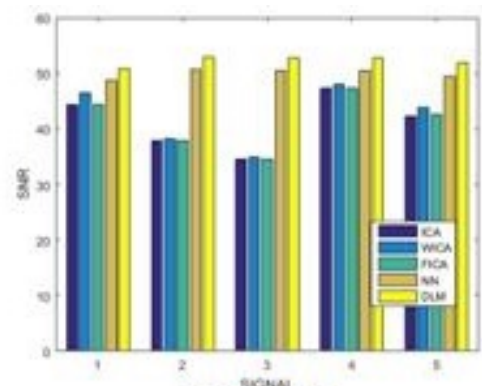

a) SNR in dB

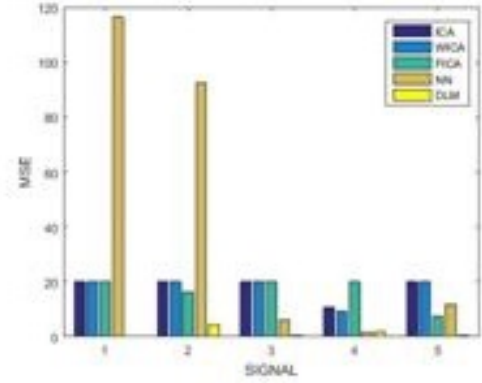

b) MSE

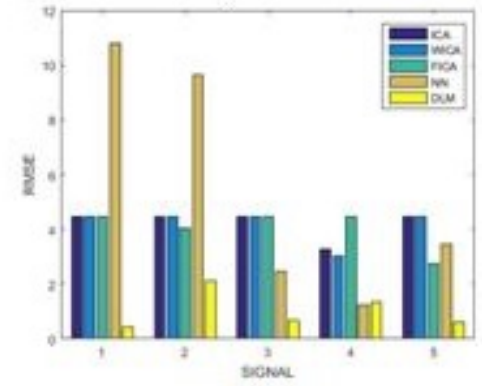

c) RMSE

FIG. 4.4. Analysis of the artefact removal in the presence of the EOG signal

ICA, WICA, FICA, NN, and DLM respectively that prove the proposed method is superior over the existing methods.

Figure 4.4.c) shows the analysis in terms of RMSE in the presence of the EOG signal. The effective method responds with the minimum value of the RMSE error. For the first signal, the RMSE values of the methods like the ICA, WICA, FICA, NN, and DLM are 4.4721, 4.4721, 4.4721, 10.7991, and 0.4280 respectively. The value of RMSE is 4.4721 for ICA, 4.4721 for WICA, 4.0376 for FICA, 9.6228 for NN, and 2.1273 for DLM, respectively for the second signal. It is clear that for the proposed DLM algorithm, the value of RMSE is minimum when compared with the existing artefact removal methods. Similarly, the analysis using the third and the fourth signal indicate that the RMSE value of the proposed DLM is less when compared with the other methods of artefact removal. Finally, for the fifth signal, the RMSE values are 4.4721, 4.4721, 4.7582, 3.4471, and 0.6141 for the artefact removal methods like the ICA, WICA, FICA, NN, and DLM respectively that prove the proposed method is superior over the existing methods.

4.5. Comparative discussion. The comparison Table 4.5 presents the comparison of the artefact removal methods with respect to the SNR, MSE, and RMSE parameters. The maximum SNR of $52.8789 \mathrm{~dB}$ is obtained using the proposed DLM method, whereas the other methods like the ICA, WICA, FICA, NN, and DLM obtained an SNR value of $44.4474 \mathrm{~dB}, 50.9866 \mathrm{~dB}, 47.9796 \mathrm{~dB}$, and $50.7035 \mathrm{~dB}$ respectively. Similarly, the MSE value for the proposed method is minimum as 0.1832 , but for the methods like the ICA, WICA, FICA, 
TABLE 4.1

Comparative discussion of the artefact removal methods

\begin{tabular}{|c|c|c|c|c|}
\hline Methods & SNR $(\mathrm{dB})$ & MSE & RMSE & Computational time (Sec) \\
\hline ICA & 44.4474 & 6.94 & 2.6345 & 12.5 \\
\hline WICA & 50.9866 & 7.1040 & 2.6653 & 11 \\
\hline FICA & 47.9796 & 7.60 & 2.75 & 9.5 \\
\hline NN & 50.7035 & 0.3088 & 0.5557 & 7 \\
\hline DLM & 52.8789 & 0.1832 & 0.428 & 6 \\
\hline
\end{tabular}

NN, and DLM, the MSE error is 6.94, 7.1040, 7.60, and 0.3088 respectively. Likewise, the minimum RMSE of 0.428 is obtained by the proposed method when compared with the other existing artefact removal methods. Also, the proposed method has the computational time of $6 \mathrm{sec}$, which is minimum than the computational time of other comparative methods.

5. Conclusion. The paper concentrates on the proposed method of artefact removal using the DLMbased NARX neural network. The proposed algorithm uses both the dragonfly optimization and LM learning algorithm for framing the DLM algorithm that trains the NARX neural network. The artefact removal used a simple subtraction method that subtracts the artefact from the ECG signal such that the ECG signals obtained are clear and is suitable for diagnosing the cardiac-related diseases. The adaptive tuning of the artefact removal is carried out using the DLM-based NARX neural network such that the proposed methods stand as an effective approach for artefact removal. The experimentation performed using the artefact signals, such as EMG, EEG, and EOG proves that the proposed method is effective when compared with the existing methods. The maximum SNR of $52.8789 \mathrm{~dB}$, a minimum error of 0.1832 , and a minimum error of 0.428 is obtained using the proposed DLM-based NARX neural network that generates the clean ECG signal. The proposed method stood as an effective method in extracting the clean signal from the artefact ECG signal. The performance of the proposed method is further increased by using recent optimization algorithms. The training speed of the proposed method also needs further improvement.

\section{REFERENCES}

[1] S. A. Imtiaz, J.Mardell, S. S.Yarahmadi, E. R.-Villegas, ECG artefact identification and removal in mHealth systems for continuous patient monitoring vol.3, no.3, pp.171 - 176, 2016.

[2] A. Kumar and M.Singh, Optimal Selection of Wavelet Function and Decomposition Level for Removal of ECG Signal Artifacts Journal of Medical Imaging and Health Informatics, Vol. 5, 138-146, 2015.

[3] S.-H.Liu, Motion artifact reduction in Electrocardiogram using adaptive filter Journal of Medical and Biological Engineering, 31(1): 67-72, 2010.

[4] M. Z. U. Rahman, R. A. Shaik, D.V. R. K. Reddy, Efficient sign based normalized adaptive filtering techniques for cancelation of artifacts in ECG signals: Application to wireless biotelemetry Signal Processing, Volume 91, Issue 2, February 2011, Pages 225-239.

[5] J.Lee, D. D. McManus, S. Merchant, and K. H. Chon,Automatic Motion and Noise Artifact Detection in Holter ECG Data Using Empirical Mode Decomposition and Statistical Approaches IEEE Transactions On Biomedical Engineering, Vol. 59, No. 6, June 2012.

[6] T. He, G. Clifford, and L. Tarassenko, Application of independent component analysis in removing artefacts from the electrocardiogram Neural Computing \& Applications, April 2006, Volume 15, Issue 2, pp 105-116.

[7] C.B. Mbachu, I.digo Victor, I. Emmanuel, and I.I. Nsionu, Filtration Of Artifacts In ECG Signal Using Rectangular Window-Based Digital Filters IJCSI International Journal of Computer Science Issues, Vol. 8, Issue 5, No 1, September 2011.

[8] G. Zhang, T. Wu, Z. Wan, Z. Song, M. Yu, D. Wang, L. Li, F. Chen, A new method to detect ventricular fibrillation from CPR artifact-corrupted ECG based on the ECG aloneBiomedical Signal Processing and Control, Volume 29, August 2016, Pages 67-75.J.

[9] S. Paul, M. R. Reddy, and V. J. Kumar, A transform domain SVD filter for suppression of muscle noise artefacts in exercise ECG's IEEE Trans. Biomed. Eng., vol. 47, no. 5, pp. 654-663, May 2000.

[10] P. Lander and E. J. Berbari, Time-frequency plane wiener filtering of the high-resolution ECG: Development and application IEEE Trans. Biomed. Eng., vol. 44, no. 4, pp. 256-265, Apr. 1997.

[11] G. Lu, J. S. Brittain, P. Holland, J. Yianni, A. L. Green, J. F. Stein, T. Z. Aziz, and S.Wang, Removing ECGnoise from surfaceEMGsignals using adaptive filtering Neurosci. Lett., vol. 462, no. 1, pp. 14-19, Oct. 2009. 
[12] D. L. Donoho, De-noising by soft-thresholding IEEE Trans. Inf. Theory, vol. 41, no. 1, pp. 613-627, May 1995.

[13] O. Sayadi And M. B. Shamsollahi, ECG denoising with adaptive bionic wavelet transform in Proc. IEEE Conf. Eng. Med. Biol. Soc., 2006, pp. 6597-6600.

[14] R. Sameni,M. B. Shamsollahi, C. Jutten, And G. D. Clifford, A nonlinear Bayesian filtering framework for ECG denoising IEEE Trans. Biomed. Eng., vol. 54, no. 12, pp. 2172-2185, Dec. 2007.

[15] Y. Kishimoto, Y. Kutsuna, And K. OGURI, Detecting motion artifact ECG noise during sleeping bymeans of a tri-axis accelerometer in Proc. IEEE Conf. Eng. Med. Biol. Soc., 2007, pp. 2669-2672.

[16] S. W. Yoon, S. D. Min, Y. H. Yun, S. LeE, AND M. LeE, Adaptive motion artifacts reduction using 3-axis accelerometer in e-textile ECG measurement system J. Med. Syst., vol. 32, no. 2, pp. 101-106, Apr. 2008.

[17] A.Agarwal, A. Singh, A. Acharyya, R. A. Shafik, S. R. Ahamed, Energy-Efficient and High-Speed Robust Channel Identification Methodology to Solve Permutation Indeterminacy in ICA for Artifacts Removal from ECG in Remote Healthcare In Proceedings of the 2013 International Symposium on Electronic System Design, pp. 52 - $56,2013$.

[18] S. A. Anapagamini, R. Rajavel, Removal of artifacts in ECG using Empirical mode decomposition In Proceedings of the 2013 International Conference on Communication and Signal Processing, pp.288 - 292, 2013.

[19] J. S. Lin, S. Y. Huang, K. W. Pan And S. H. LiU, A physiological signal monitoring system based on an SoC platform and wireless network technologies in homecare technology J. Med. Bio. Eng., 29: 47-51, 2009.

[20] S. Kim, H. Nakamura, T. Yoshida, M. Kishimoto, Y. Imai, N. Matsuki, T. Ishikawa and T. Yamaguchi, Development of a wearable system module for monitoring physical and mental workload Telemed. J. E-Health, 14: 939-945, 2008.

[21] S. MiRJAlili, Dragonfly algorithm: a new meta-heuristic optimization technique for solving single-objective, discrete, and multi-objective problems Neural Computing and Applications, Vol. 27, No. 4, pp. 1053-1073, May 2016.

[22] J. M. P. Menezes JR AND G. A. BARReto, Long-term time series prediction with the NARX network: An empirical evaluation Neurocomputing, vol.71, no.16-18, pp.3335-3343, October 2008.

[23] Gaidhane, H. Vilas, V, Singh, Y. V. Hote, and M. Kumar, New approaches for image compression using neural network Journal of Intelligent Learning Systems and Applications, vol.3, no.04, pp.220-229, 2011.

[24] A. Jafarifarmand, M. A. Badamchizadeh, Artifacts removal in EEG signal using a new neural network enhanced adaptive filter Neurocomputing, vol.103, pp.222-231,1 March 2013.

[25] Y. LI, Automatic removal of the eye blink artifact from EEG using an ICA-based template matching approach Physiological Measuremets, vol.27, pp.425-36, 2006.

[26] P. Mishra And S. K. Singla, Artifact Removal from Biosignal using Fixed Point ICA Algorithm for Pre-processing in Biometric Recognition vol.13, no.1, Jan 2013.

[27] B. Azzerboni, M. Carpentien, E.L. Foresta, And E C. Morabito, Neural-ICA and Wavelet Transform for Artifacts Removal in surface EMG In Proceedings of IEEE International Joint Conference on Neural Networks, Budapest, Hungary, 2004.

[28] S. PoungPonsRi And X.-HuAYu, An adaptive filtering approach for electrocardiogram (ECG) signal noise reduction using neural networks Neurocomputing, vol. 117, pp. 206-213, 2013.

[29] P. Amani, M. Kinl, And A. Robertsson, NARX-based Multi-step Ahead Response Time Prediction for Database Servers In proceedings of 11th International Conference on Intelligent Systems Design and Applications (ISDA), Cordoba, Spain, 2011.

[30] Z. Boussaada, O. Curea, A. Remaci, H. Camblong, and N. M. Bellaaj, A Nonlinear Autoregressive Exogenous (NARX) Neural Network Model for the Prediction of the Daily Direct Solar Radiation Energies, vol. 11, no. 3, 2018.

[31] E. Diaconescu, The use of NARX Neural Networks to predict Chaotic Time Series WSEAS Transactions on Computer Research, vol. 3, no. 3, pp. 182-191, March 2008.

[32] H. He, X. YAN AND W.WeI, Meridian ECG Information Transmission System Modeling Using NARX Neural Network In proceedings of IEEE/ACIS 15th International Conference on Computer and Information Science (ICIS), Okayama, Japan, 2016.

[33] A. Ratre AND V. PANKAJAKSHAN Tucker visual search-based hybrid tracking model and Fractional Kohonen Self-Organizing Map for anomaly localization and detection in surveillance videos The Imaging Science Journal, pp. 1-16, 2017.

[34] A. V. Dhumane AND R.S. PRASAD Multi-objective fractional gravitational search algorithm for energy efficient routing in IoT Wireless Networks, pp. 1-15, 2017.

[35] S. Nipanikar, V H. Deepthi, And N. Kulkarni, A sparse representation based image steganography using Particle Swarm Optimization and wavelet transform 2017.

[36] P.M. Shelke And R. S. PRASAD An improved anti-forensics JPEG compression using Least Cuckoo Search algorithmThe Imaging Science Journal, vol. 66, no. 3, pp. 169-183, 2018.

Edited by: P. Vijaya

Received: Dec 7, 2019

Accepted: Jun 23, 2020 